\title{
Pimecrolimus Reduces Eosinophil Activation Associated with Calcium Mobilization
}

\author{
Douglas A. Plager ${ }^{\mathrm{a}}$ Susan A. Henke ${ }^{\mathrm{a}}$ Yoshinori Matsuwaki $^{\mathrm{a}}$ Arvind Madaan $^{\mathrm{a}}$ \\ Diane L. Squillace $^{a}$ Ross A. Dierkhising ${ }^{b}$ Hirohito Kita $^{a}$

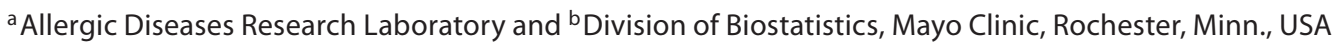

\section{Key Words}

Alternaria alternate $\cdot$ Atopic dermatitis $\cdot$ Calcineurin

inhibitor $\cdot$ Eosinophil $\cdot$ Fungus $\cdot$ Pimecrolimus

\begin{abstract}
Background: Pimecrolimus is a calcineurin inhibitor that inhibits $T$ cell and mast cell activation and effectively treats atopic dermatitis. However, its effects on eosinophils, a cell type implicated in allergic disease pathology, are unknown. Therefore, we examined the effects of pimecrolimus on eosinophil superoxide anion production, degranulation and survival. Methods: Purified eosinophils from normal or atopic donors were incubated with serial dilutions of pimecrolimus ( $\mu \mathrm{M}$ to $\mathrm{nM})$ and then stimulated with platelet activating factor (PAF), interleukin 5 (IL5), secretory immunoglobulin A $(\mathrm{s} \lg \mathrm{A})$ or Alternaria alternata (Alt) fungus extract. Eosinophil activation was monitored by cytochrome $c$ reduction resulting from superoxide anion production and by a 2-site immunoassay for eosinophil-derived neurotoxin (EDN) in cellular supernatants, as a marker of degranulation. Eosinophil survival was measured by propidium iodide exclusion using flow cytometry after 4 days in culture. Results: Normal and atopic eosinophil superoxide anion production induced by PAF, and associated with increased intracellular calcium, was inhibited up to $37 \%$ with $1 \mu \mathrm{M}$ pimecrolimus. However, superoxide anion production induced by IL5 and slgA was not consistently inhibited. EDN release, which ultimately de-
\end{abstract}

pends on calcium, was inhibited about $30 \%$ with PAF, IL5 and slgA stimulation for normal and atopic donor eosinophils. Furthermore, calcium-dependent Alt-induced EDN release was inhibited up to $49 \%$ with nanomolar pimecrolimus. Finally, increased eosinophil survival promoted by IL5 and slgA was not influenced by pimecrolimus. Conclusion: Pimecrolimus moderately inhibits eosinophil superoxide anion production and EDN release associated with calcium mobilization, which may contribute to its efficacy in treating atopic dermatitis.

Copyright $\odot 2009$ S. Karger AG, Basel

\section{Introduction}

Atopic dermatitis is a common, chronic skin disease characterized by extremely pruritic eczematous lesions that often become infected or lichenified with scratching [1]. The prevalence of childhood atopic dermatitis in the United States and other Westernized societies is around $10 \%[2,3]$, and its prevalence and that of the other allergic diseases (asthma and allergic rhinitis) appear to have risen over the past several decades [4]. Atopic dermatitis often continues into adulthood $[1,5,6]$ or it may transition

D.A.P. and H.K. were recipients of funds from the Novartis Pharmaceutical Corporation in support of the research reported here.

\section{KARGER \\ Fax +41613061234 E-Mail karger@karger.ch} www.karger.com
Correspondence to: Dr. Douglas A. Plager

Department of Dermatology, Mayo Clinic

Guggenheim 4-94, 200 First Street Southwest

Rochester, MN 55905 (USA)

Tel. +1 507284 9931, Fax +1 507284 5045, E-Mail plager.douglas@mayo.edu 
into allergic airway disease (referred to as the 'atopic march'). Severe allergic disease can have a substantial socioeconomic impact on the patient and their family [7]. Topical corticosteroids, oral antihistamines and adjunctive antimicrobial agents (e.g. antibiotics) have been the primary therapeutic treatments for atopic dermatitis for several decades, with more potent immunosuppressive therapies used for refractory disease [8]. Unfortunately, numerous side effects may arise with the prolonged use of topical corticosteroids, skin atrophy being the most notable.

More recently, topical calcineurin inhibitors [pimecrolimus cream 1\% (Elidel ${ }^{\circledR}$, Novartis Pharmaceutical Corp., East Hanover, N.J., USA) and tacrolimus (Protopic ${ }^{\circledR}$, Astellas Pharma Inc., Tokyo, Japan)] have demonstrated their therapeutic benefit in treating mild to severe atopic dermatitis, and transient skin burning or irritation is the only currently recognized common adverse reaction $[8$, 9]. Each of these macrolide compounds binds to macrophilin-12 and the resulting complex then binds to the cytosolic and calcium/calmodulin-dependent phosphatase, calcineurin. This inhibits the ability of calcineurin to dephosphorylate the inactive cytosolic form of nuclear factor of activated $\mathrm{T}$ cells, ultimately inhibiting its translocation to the nucleus and its induction of type 1 [interleukin 2 (IL2), IFN $\gamma, \mathrm{TNF} \alpha$ ], type 2 (IL4, IL5) and regulatory (IL10) cytokines [10]. Inhibited cytokine production from both cutaneous T cells [11] and mast cells [12] appears to contribute to the therapeutic efficacy of the calcineurin inhibitiors $[13,14]$. However, inhibition of other cellular responses, such as FceRI-mediated mast cell degranulation and secretion, may also contribute to their anti-inflammatory properties [15].

Eosinophil accumulation in affected tissues is characteristic of atopic disease and is closely associated with the severity of atopic dermatitis [16-19]. Delayed eosinophil apoptosis, deposition of eosinophil granule proteins and superoxide anion production each appear to contribute to disease pathology [19-21]. More recent studies also suggest that the eosinophil functions more broadly in allergic disease pathophysiology [22]. Tacrolimus [23-25] and corticosteroids [26] can directly modulate eosinophil activity such as cytokine production or eosinophil survival; however, it remains unknown whether pimecrolimus can directly influence eosinophil activity. In addition, some studies suggest that tacrolimus and corticosteroids inhibit dendritic cell activity and/or viability much more so than pimecrolimus, so the potential for differing effects on eosinophil activity among these various therapeutic compounds exists. Therefore, because the eosino- phil is implicated as a major effector cell in allergic disease, we sought to determine the effect of pimecrolimus on eosinophil effector functions and survival.

\section{Materials and Methods}

\section{Eosinophil Isolation and Donors}

Human eosinophils were purified from $90 \mathrm{ml}$ peripheral blood to greater than $94 \%$ using Percoll density gradient centrifugation followed by negative magnetic cell separation [27]. Donors included individuals with ('atopic') or without ('normal') a physician-diagnosed history of allergic disease (atopic dermatitis/eczema, asthma, allergic rhinitis/hay fever). No donors received systemic immunosuppressants within 4 weeks of their blood donation and none had confounding concurrent disease. Informed consent was provided by all donors under an institutional review board-approved protocol.

\section{Eosinophil Superoxide Production and Degranulation}

In 96-well plates, platelet activating factor (PAF; $1 \mu \mathrm{M})$, IL-5 (10 ng/ml), immobilized secretory immunoglobulin A (sIgA) coated on polystyrene beads (Polysciences Inc.; $10 \mu \mathrm{m}$ diameter) or on the plate wells, and Alternaria alternata (Alt) fungus extract (Greer Laboratories; $50 \mu \mathrm{g} / \mathrm{ml}$ ) were used to activate purified eosinophils (50,000/well) in the absence or presence of varying concentrations of pimecrolimus (30-60 min preincubation with pimecrolimus at room temperature). Purified eosinophils in medium alone (HBSS, $10 \mathrm{mM}$ HEPES, $0.01 \%$ gelatin) or activated with phorbol myristate acetate (PMA, $1 \mathrm{ng} / \mathrm{ml}$ ) were used as negative and positive controls, respectively. Superoxide anion production was assessed via superoxide dismutase-inhibitable reduction of cytochrome $\mathrm{c}(100 \mu \mathrm{M})$ by measuring absorbance at 550 $\mathrm{nm}$ [28]. Four hours after initiation of superoxide production, 96well plates were spun at $175 g$ for 5 min and supernatant fluid was collected. To assess degranulation, eosinophil-derived neurotoxin (EDN) levels in the supernatants were measured using a double-antibody competition radioimmunoassay [29].

\section{Intracellular Calcium}

Purified eosinophils at $2 \times 10^{6}$ cells $/ \mathrm{ml}$ were incubated with $3 \mathrm{mM}$ indo-1/AM (Molecular Probes, Eugene, Oreg., USA) in HBSS, $10 \%$ alpha calf serum, $10 \mathrm{mM}$ HEPES for $30 \mathrm{~min}$ at $37^{\circ} \mathrm{C}$. After washing twice, cells were resuspended in HBSS, $0.1 \%$ HSA, $10 \mathrm{mM}$ HEPES at $2 \times 10^{6} \mathrm{cells} / \mathrm{ml}$. When appropriate, eosinophils were pretreated with $1 \mathrm{nM}$ pimecrolimus for $1 \mathrm{~h}$ on ice. Eosinophils were then stimulated with PAF $(1 \mu \mathrm{M})$ or Alt $(75 \mu \mathrm{g} / \mathrm{ml})$, and fluorescence was analyzed over time by a FACS analyzer equipped with an ion-argon laser. Intracellular calcium was monitored by the fluorescence ratio of calcium-bound indo-1 emission (401 $\mathrm{nm})$ and free indo- 1 emission $(475 \mathrm{~nm})$.

\section{Eosinophil Survival}

Eosinophil survival was measured following the method of Bankers-Fulbright et al. [30]. Purified eosinophils were incubated in medium alone (RPMI $1640+10 \%$ alpha calf serum) or in the presence of varying concentrations of pimecrolimus at $37^{\circ} \mathrm{C}, 5 \%$ $\mathrm{CO}_{2}$ for $30 \mathrm{~min}$. Additional medium, IL5 (75 pg/ml), or soluble $\operatorname{sigA}(10 \mu \mathrm{g} / \mathrm{ml})$ was added and cells were incubated at $37^{\circ} \mathrm{C}, 5 \%$ 


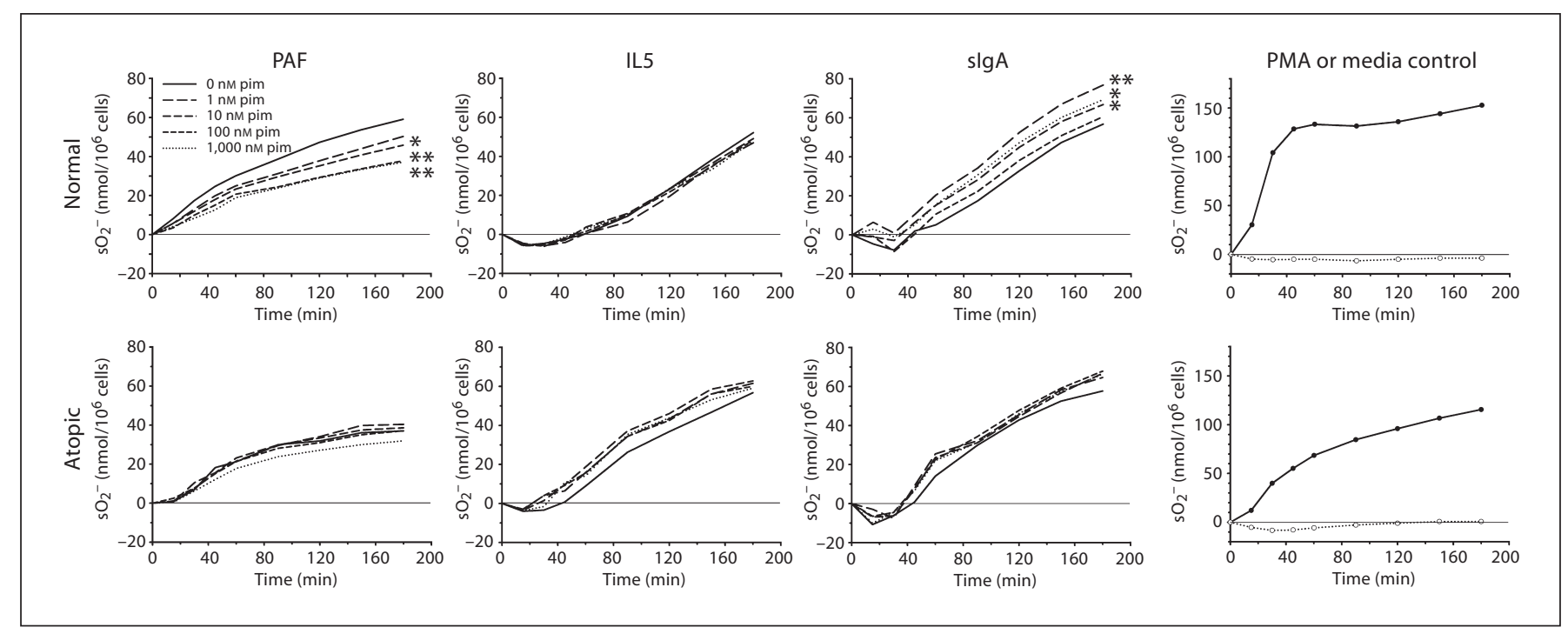

Fig. 1. Effect of pimecrolimus on eosinophil superoxide production. Mean superoxide anion $\left(\mathrm{sO}_{2}^{-}\right)$production by purified eosinophils from normal $(n=13)$ or atopic donors $(n=9$ or 10$)$ is shown following stimulation with PAF, IL5 or immobilized sIgA after pre-incubating eosinophils with no pimecrolimus (i.e. $0 \mathrm{nM}$ pim; solid line) or with pimecrolimus at $1 \mathrm{~nm}$ (long dashes), $10 \mathrm{~nm}$ (me- dium dashes), $100 \mathrm{~nm}$ (short dashes) or 1,000 nM (dotted). Positive (PMA, closed circles) and negative (medium alone, open circles) control responses are shown $(n=11$ for normals and $n=4$ for atopics). Significant changes in superoxide anion production relative to stimuli alone (i.e. $0 \mathrm{nM}$ pimecrolimus) at the 180 -min time point are indicated by asterisks. ${ }^{*} \mathrm{p}<0.05 ;{ }^{* *} \mathrm{p}<0.01$.
$\mathrm{CO}_{2}$ for 4 days. Propidium iodide staining of dead cells was assessed by flow cytometry, and data, where survival induced by stimuli alone was at least twice that of survival in medium alone, were analyzed further.

\section{Statistical Analyses}

Repeated measures models were used to estimate the effect of pimecrolimus level on eosinophil EDN release and eosinophil survival in atopic and normal subjects separately. Individual models were fit for each stimuli (PAF, IL5, sIgA or Alt). A compound symmetric correlation structure (i.e. equal correlations) was used in these models to estimate the correlations between pimecrolimus levels within a patient. The 'sandwich' estimator of the covariance matrix of the parameters was used for inferences [31]. The 'sandwich' estimator is asymptotically consistent for the true parameter covariance matrix even if the within-patient correlation matrix is inappropriately specified. The same type of repeated measures models were estimated to measure the effect of pimecrolimus concentration on superoxide production. Separate models were fit for atopic and normal donors at the 180-min time point for PAF, IL5 and SIgA stimuli.

\section{Results}

Two common measures of human eosinophil activation are extracellular superoxide anion production and EDN release from their specific secondary granules.
These eosinophil responses appear important to allergic disease pathology and can be induced by distinct stimuli, such as the lipid mediator PAF, the cytokine IL5 and the immunoglobulin complex sIgA. Topical $1 \%$ pimecrolimus, which is used to treat atopic dermatitis, corresponds to a $1 \mathrm{mM}$ pimecrolimus concentration, and penetration studies in minipig skin indicate that concentrations of $0.1 \mu \mathrm{M}$ pimecrolimus or greater can be expected to reach the upper dermis, depending on the integrity of the skin [32]. The influence of pimecrolimus in the micromolar to nanomolar concentration range on eosinophil superoxide anion production induced by PAF, IL5 and $\operatorname{sIg} \mathrm{A}$ is shown in figure 1. Because eosinophil responses can vary with the atopic status of the donor, data for normal and atopic donors were analyzed separately. Only PAF-induced superoxide anion production for normal donors showed a significant and dose-dependent inhibition by pimecrolimus at the $180 \mathrm{~min}$ endpoint (fig. 2). The effects of pimecrolimus on eosinophil EDN release occurring under the experimental conditions for measuring superoxide anion production were also assessed (fig. 3). In the majority of these cases, EDN release was partially inhibited at the higher pimecrolimus concentrations. Only sIgA-stimulated superoxide anion production (fig. 2) and EDN release (fig. 3) from normal donor 

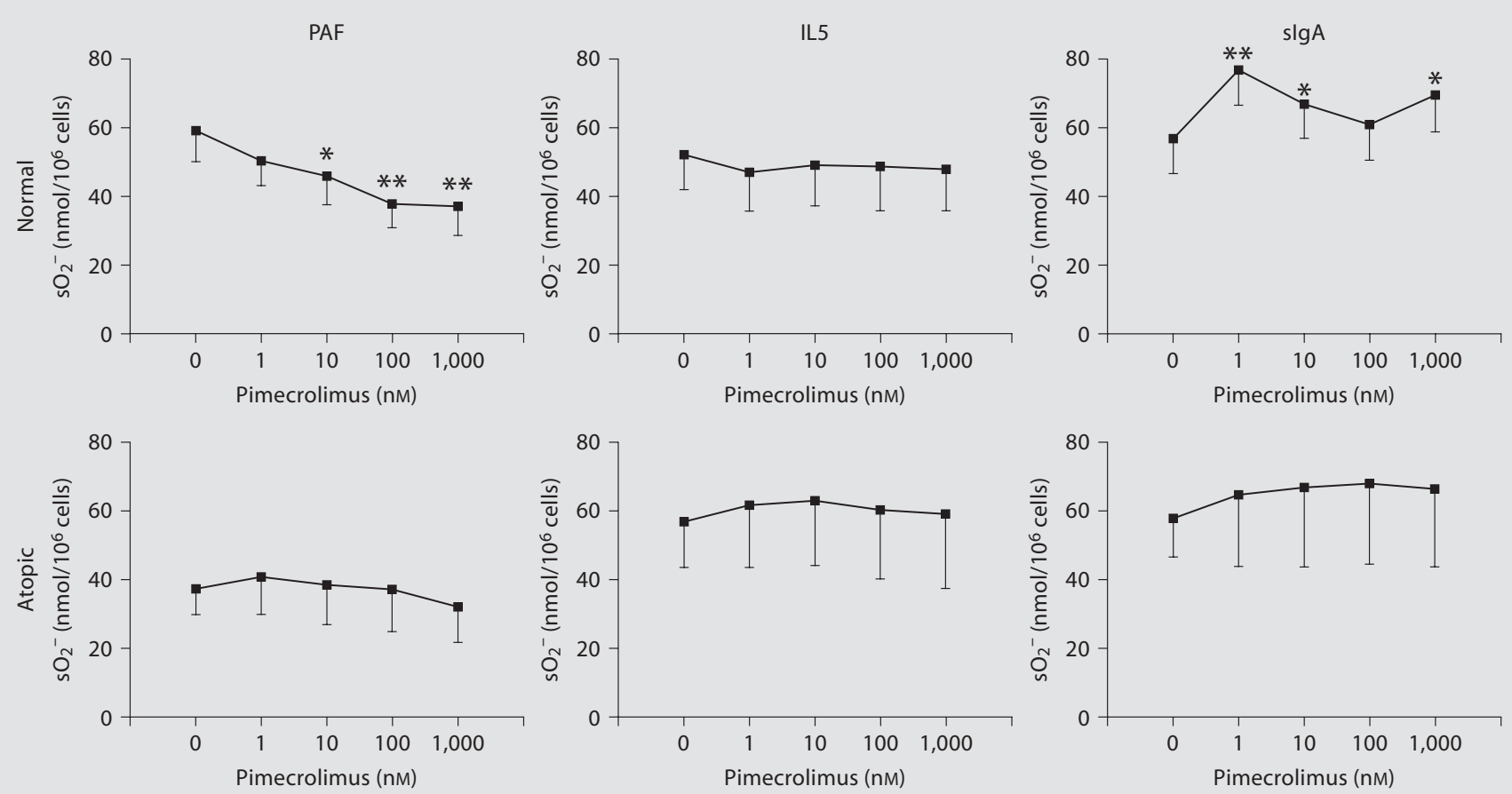

Fig. 2. Pimecrolimus dose effect on endpoint eosinophil superoxide production. Mean superoxide anion $\left(\mathrm{sO}_{2}^{-}\right)$production after incubating for $180 \mathrm{~min}$ in the absence $(0 \mathrm{nM})$ and presence of increasing concentrations of pimecrolimus (1 to $1,000 \mathrm{nM}$ ) are shown. Values are from figure 1 following stimulation of normal or atopic donor eosinophils with PAF, IL5 or immobilized sIgA. Significant changes in superoxide anion production relative to stimuli alone (i.e. $0 \mathrm{nM}$ pimecrolimus) are indicated by asterisks. ${ }^{*} \mathrm{p}<0.05 ;{ }^{* *} \mathrm{p}<0.01$. Standard errors of the means are indicated by unidirectional error bars. eosinophils were significantly increased. However, this increased activation with pimecrolimus did not appear to occur in a dose-dependent manner, as only the lowest concentration of pimecrolimus tested here ( $1 \mathrm{nM})$ resulted in both significantly increased superoxide anion production and EDN release.

Environmental fungal triggers, including that of $\mathrm{Al}$ ternaria alternata (Alt), have long been associated with exacerbation of atopic disease. Recent evidence from our laboratory indicates that Alt-derived products can directly activate eosinophils [33]. Therefore, the influence of pimecrolimus on Alt extract-induced EDN release from normal and atopic donor eosinophils was also tested (fig. 3). Similar to that for the other stimuli (PAF, IL5 and sIgA), Alt-induced EDN release was also inhibited by pimecrolimus. However, inhibition of Alt-induced EDN release was observed even at subnanomolar pimecrolimus concentrations (fig. 3). Because Alt extract components compromise the superoxide anion production as- say, no superoxide anion production results with Alt extract stimulation were obtained.

Inhibition of eosinophil activation by PAF and Alt occurred most consistently and in a dose-dependent manner. Furthermore, eosinophil activation by these 2 stimuli involves rapid increase in intracellular calcium [33, 34]. Thus, to test whether pimecrolimus impacts the calcium flux in PAF- and Alt-activated eosinophils, intracellular calcium levels were monitored using a calciumsensitive fluorescent compound (fig. 4). No change in the magnitude of the intracellular calcium response was observed.

As a final measure of an eosinophil property associated with atopic disease, increased survival, which is induced by IL5 and SIgA, but not PAF, was assessed in the presence of increasing concentrations of pimecrolimus for eosinophils from normal and atopic donors (fig. 5). No consistent change in eosinophil survival occurred in the presence of pimecrolimus. 

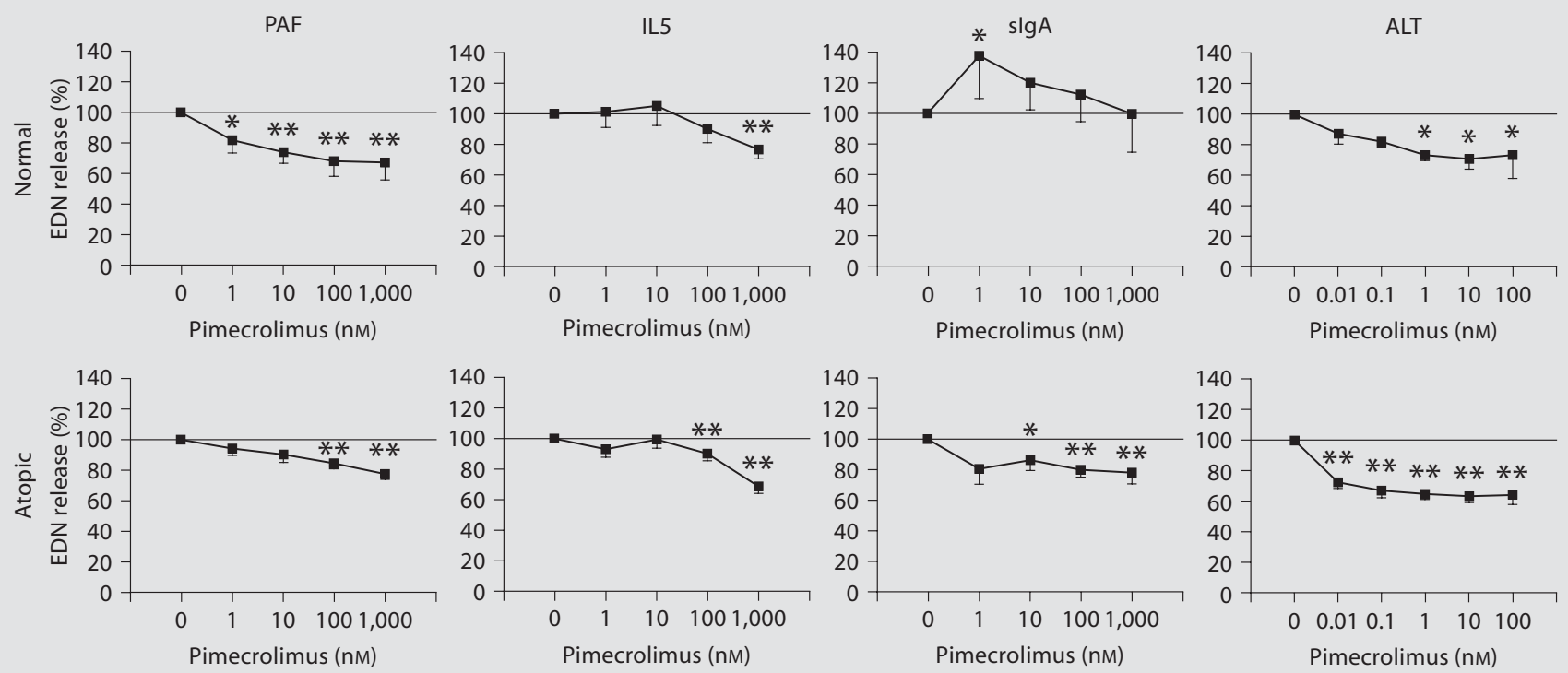

Fig. 3. Effect of pimecrolimus on eosinophil EDN release. Cellular supernatant collected at the end of superoxide anion $\left(\mathrm{sO}_{2}^{-}\right)$production experiments (fig. 1 ) were assayed for EDN content ( $\mathrm{n}=11$ for normals, $n=4$ for atopics). Data are presented as percent EDN release relative to that induced by stimuli (PAF, IL5 or immobilized $\operatorname{sIg} \mathrm{A}$ ) alone. In separate experiments (ALT extract complicates measurement of superoxide anion production), EDN release from purified eosinophils ( $\mathrm{n}=3$ for normals, $\mathrm{n}=5$ for atopics) induced by ALT fungal extract was measured. In all instances, the mean percent EDN release in the presence of medium alone (i.e. with no stimulant) was within the range of 15 to $28 \%$. Significant changes in percent EDN release relative to stimuli alone (i.e. $0 \mathrm{nM}$ pimecrolimus) are indicated by asterisks. ${ }^{*} \mathrm{p}<0.05 ;{ }^{* *} \mathrm{p}<0.01$. Standard errors of the means are indicated by unidirectional error bars.

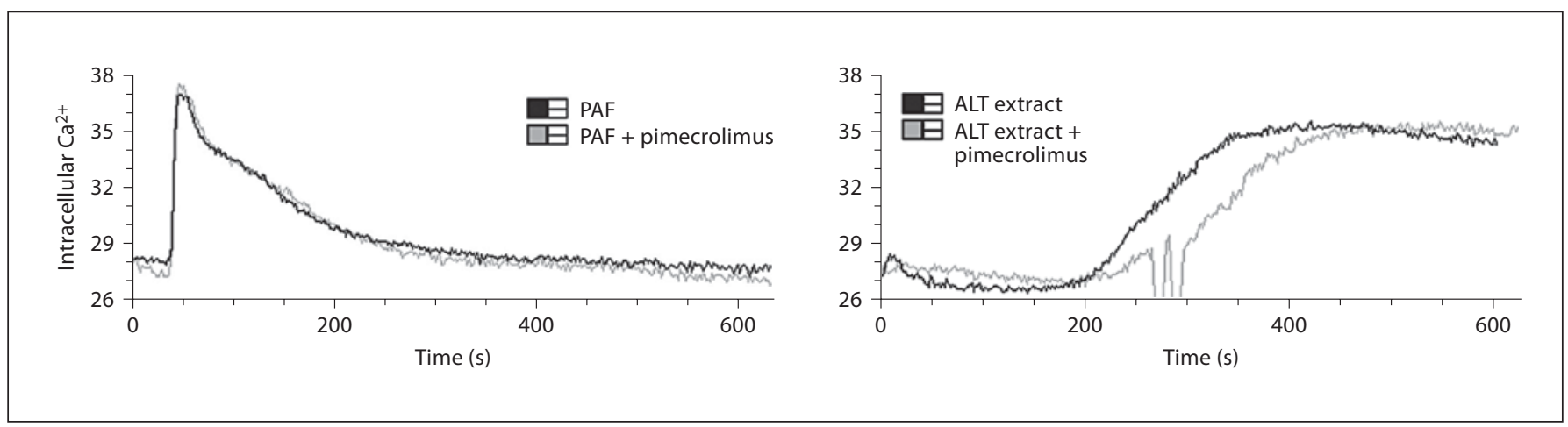

Fig. 4. Intracellular calcium flux induced by PAF and ALT in the absence and presence of pimecrolimus. Purified eosinophils loaded with a fluorescent calcium indicator (indo-1/AM) were stimulated with $1 \mu \mathrm{M}$ PAF or $75 \mu \mathrm{g} / \mathrm{ml}$ ALT fungal extract (black lines). In a second round of experiments, eosinophils were pretreated with $1 \mathrm{nM}$ pimecrolimus for $1 \mathrm{~h}$ on ice before PAF or ALT stimulation (gray lines). Eosinophils were all from a single individual, and the sharp fluctuations in the ALT extract + pimecrolimus trace are artificial. 
Fig. 5. Effect of pimecrolimus on eosinophil survival. Purified eosinophils were preincubated for $30 \mathrm{~min}$ without or with varying concentrations of pimecrolimus [ $\mathrm{n}=11$ (IL5) or 7 (sIgA) for normals, $\mathrm{n}=7$ for atopics]. IL5 $(75 \mathrm{pg} / \mathrm{ml})$ or soluble sIgA $(10 \mu \mathrm{g} / \mathrm{ml})$ was then added and cell survival was measured 4 days later. Data are presented as percent survival relative to that induced by stimuli (IL5 or sIgA) alone. In all instances, the mean percent survival in the presence of medium alone (i.e. with no stimulant) was within the range of 17 to $25 \%$. Significant changes in percent survival relative to stimuli alone (i.e. $0 \mathrm{~nm}$ pimecrolimus) are indicated by asterisks. ${ }^{*} \mathrm{p}<0.05 ;{ }^{* *} \mathrm{p}<0.01$. Standard errors of the means are indicated by unidirectional error bars.
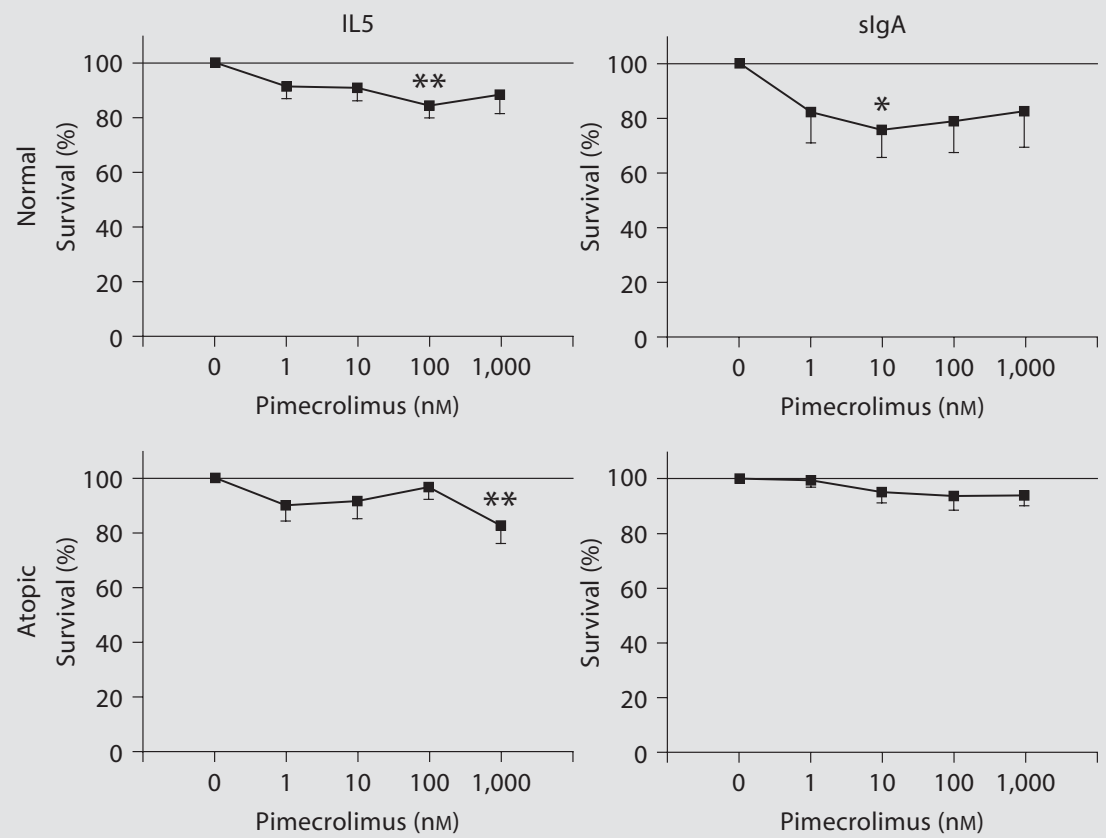

\section{Discussion}

Eosinophils are implicated as key effector cells in allergic disease, such as atopic dermatitis, and additional roles for eosinophils in allergic disease pathology have been suggested [22]. The recently developed calcineurin inhibitors tacrolimus and pimecrolimus have shown their effectiveness in treating atopic dermatitis. However, the direct effect of pimecrolimus on eosinophil function has not been reported previously. Here we show that eosinophil activation induced by calcium mobilizing stimuli, such as PAF and Alt extract, was significantly inhibited by nanomolar concentrations of pimecrolimus. Eosinophil degranulation, which ultimately depends on calcium, further appears to be generally inhibited by $1 \mu \mathrm{M}$ pimecrolimus. However, stimulus-dependent increase in intracellular calcium was not altered by pimecrolimus, suggesting that pimecrolimus affects an event (or events) downstream of activation-induced intracellular calcium increase.

Various stimuli can activate human eosinophils, including some that induce a rapid increase in intracellular calcium, like PAF and Alt extract $[33,34]$. Upon activation, extracellular superoxide anion production and release of cationic secondary granule proteins, including EDN, are 2 common eosinophil effector functions that appear to contribute to allergic disease pathology. Therefore, we assessed the effect of pimecrolimus on these 2 effector functions. PAF-induced superoxide anion production by eosinophils from normal donors was inhibited up to $37 \%$ by pimecrolimus in a dose-dependent manner (fig. 1, 2). For atopic donors, PAF-induced superoxide anion appeared to increase slightly from baseline with the addition of low concentrations of pimecrolimus; however, similar to that for normal donor eosinophils, superoxide anion production was subsequently reduced in a dose-dependent manner, albeit only slightly (up to $14 \%$ when compared to measurements with no pimecrolimus; fig. 1, 2). Notably, baseline superoxide anion production from the atopic donors' eosinophils in response to PAF stimulation was relatively diminished compared with that from the normal donors ( 37 and $59 \mathrm{nmol}$, respectively). Perhaps the subdued responsiveness to PAF of the atopic donors' eosinophils contributed to the limited inhibition of superoxide anion production by pimecrolimus in this instance. Regardless, eosinophil superoxide anion production induced by IL5 and sIgA, which do not induce a rapid increase in intracellular calcium [33], were not inhibited or increased in a consistent dose-dependent manner (fig. 1,2).

After measurement of superoxide anion production, cellular supernatant from each well was collected and tested for EDN concentration as a measure of stimuli-in- 
duced eosinophil degranulation. Similar to the effect of pimecrolimus on PAF-induced superoxide production, PAF-induced EDN release was significantly inhibited at relatively low pimecrolimus concentrations for normal donor eosinophils and also at higher pimecrolimus concentrations for atopic donor eosinophils (fig. 3). While IL5 and sIgA do not rapidly mobilize intracellular calci$\mathrm{um}$, their induction of EDN release from eosinophils ultimately depends on calcium $[35,36]$. This EDN release was also significantly inhibited (about 25\%) at higher pimecrolimus concentrations in all instances, except for sIgA stimulation of normal donor eosinophils (fig. 3).

Previous work from our laboratory showed that certain environmental fungi induce EDN release from eosinophils and that this process involves an increase in intracellular calcium [33]. Extract from Alternaria alternata was one of the eosinophil-activating mixtures. Exposure to Alternaria is a risk factor for symptom exacerbation [37] and respiratory arrest in asthmatic patients [38], and it is a common IgE-reactive allergen for atopic dermatitis patients [39]. Given the apparent predilection of pimecrolimus for inhibiting eosinophil activation associated with calcium mobilization and its known mechanism for inhibiting calcium/calmodulin-dependent calcineurin activity, the effect of pimecrolimus on calciumdependent Alt-induced EDN release was also tested. Interestingly, pimecrolimus inhibited Alt-induced EDN release to a degree (36\%) comparable to that for PAF-induced EDN release and at similar or lower pimecrolimus concentration (fig. 3). If one considers the level of spontaneous EDN release, approximately $20 \%$ on average, pimecrolimus inhibited up to $49 \%$ of non-spontaneous, Alt-induced EDN release. Thus, pimecrolimus, as well as EGTA and pertussis toxin [33], can substantially inhibit Alt-induced eosinophil EDN release.

Because PAF and Alt extract induce rapid increases in intracellular calcium, we tested whether pimecrolimus acts by inhibiting calcium mobilization in eosinophils. As shown in figure 4, pimecrolimus did not appreciably alter the increase in intracellular calcium induced by PAF or Alt. Therefore, in accord with the previously reported mechanism of action, pimecrolimus appears to function in eosinophils downstream of initial calcium mobilization. Whether this involves binding of pimecrolimus to macrophilin-12, reduced calcineurin activity and/or inhibition of a downstream process directly dependent on calcium mobilization remains to be determined.

With regard to increased eosinophil survival induced by IL5 and SIgA (PAF does not induce eosinophil survival), no appreciable dose-dependent influence of pimecrolimus was observed (fig. 5). A lack of inhibition by pimecrolimus is consistent with the calcium mobilization-independent mechanism of enhanced eosinophil survival induced by these stimuli $[33,36]$.

In summary, pimecrolimus does not appear to influence calcium mobilization-independent eosinophil responses. However, it does moderately inhibit eosinophil superoxide anion production and EDN release associated with calcium mobilization via a mechanism apparently working downstream of initial intracellular calcium influx. This includes a nearly $50 \%$ inhibition of nonspontaneous EDN release triggered by a relevant environmental allergen, Alternaria fungi. This inhibition of eosinophil responses associated with calcium mobilization may contribute to the efficacy of pimecrolimus in treating atopic dermatitis.

\section{Acknowledgements}

Financial support was provided by Novartis Pharmaceuticals Corporation, the Mayo Foundation and the Dr. Smith H. and Lucille Gibson Postdoctoral Research Fellowship in Dermatology (to D.A.P.).

\section{References}

1 Leung DY, Bieber T: Atopic dermatitis. Lancet 2003;361:151-160.

-2 Laughter D, Istvan JA, Tofte SJ, Hanifin JM: The prevalence of atopic dermatitis in Oregon schoolchildren. J Am Acad Dermatol 2000;43:649-655.

-3 Worldwide variation in prevalence of symptoms of asthma, allergic rhinoconjunctivitis, and atopic eczema: ISAAC. The International Study of Asthma and Allergies in Childhood (ISAAC) Steering Committee. Lancet 1998;351:1225-1232.
4 Diepgen TL: Is the prevalence of atopic dermatitis increasing?; in Williams HC (ed): Atopic Dermatitis: The Epidemiology, Causes and Prevention of Atopic Eczema. Cambridge, Cambridge University Press, 2000, pp 96-109.

5 Linna O, Kokkonen J, Lahtela P, Tammela O Ten-year prognosis for generalized infantile eczema. Acta Paediatr 1992;81:1013-1016.
6 Kissling S, Wüthrich B: Sites, types of manifestations and micromanifestations of atopic dermatitis in young adults: a personal follow-up 20 years after diagnosis in childhood (in German). Hautarzt 1994;45:368-371.

-7 Carroll CL, Balkrishnan R, Feldman SR, Fleischer AB Jr, Manuel JC: The burden of atopic dermatitis: impact on the patient, family, and society. Pediatr Dermatol 2005; 22:192-199. 
8 Akdis CA, Akdis M, Bieber T, Bindslev-Jensen C, Boguniewicz M, Eigenmann P, Hamid Q, Kapp A, Leung DY, Lipozencic J, Luger TA, Muraro A, Novak N, Platts-Mills TA, Rosenwasser L, Scheynius A, Simons FE, Spergel J, Turjanmaa K, Wahn U, Weidinger $S$, Werfel T, Zuberbier T: Diagnosis and treatment of atopic dermatitis in children and adults: European Academy of Allergology and Clinical Immunology/American Academy of Allergy, Asthma and Immunology/PRACTALL Consensus Report. J Allergy Clin Immunol 2006;118:152-169.

-9 Stander S, Stander H, Seeliger S, Luger TA, Steinhoff M: Topical pimecrolimus and tacrolimus transiently induce neuropeptide release and mast cell degranulation in murine skin. Br J Dermatol 2007;156:10201026.

10 Hultsch T, Kapp A, Spergel J: Immunomodulation and safety of topical calcineurin inhibitors for the treatment of atopic dermatitis. Dermatology 2005;211:174-187.

11 Nghiem P, Pearson G, Langley RG: Tacrolimus and pimecrolimus: from clever prokaryotes to inhibiting calcineurin and treating atopic dermatitis. J Am Acad Dermatol 2002;46:228-241.

12 Zuberbier T, Chong SU, Grunow K, Guhl S, Welker P, Grassberger M, Henz BM: The ascomycin macrolactam pimecrolimus (Elidel, SDZ ASM 981) is a potent inhibitor of mediator release from human dermal mast cells and peripheral blood basophils. J Allergy Clin Immunol 2001;108:275-280.

-13 Simon D, Vassina E, Yousefi S, Kozlowski E, Braathen LR, Simon HU: Reduced dermal infiltration of cytokine-expressing inflammatory cells in atopic dermatitis after shortterm topical tacrolimus treatment. J Allergy Clin Immunol 2004;14:887-895.

-14 Simon D, Vassina E, Yousefi S, Braathen LR, Simon HU: Inflammatory cell numbers and cytokine expression in atopic dermatitis after topical pimecrolimus treatment. Allergy 2005;60:944-951

-15 Grassberger M, Baumruker T, Enz A, Hiestand P, Hultsch T, Kalthoff F, Schuler W, Schulz M, Werner FJ, Winiski A, Wolff B, Zenke G: A novel anti-inflammatory drug, SDZ ASM 981, for the treatment of skin diseases: in vitro pharmacology. Br J Dermatol 1999;141:264-273.
16 Leiferman KM, Ackerman SJ, Sampson HA, Haugen HS, Venencie PY, Gleich GJ: Dermal deposition of eosinophil-granule major basic protein in atopic dermatitis: comparison with onchocerciasis. N Engl J Med 1985;313: 282-285.

17 Ott NL, Gleich GJ, Peterson EA, Fujisawa T, Sur S, Leiferman KM: Assessment of eosinophil and neutrophil participation in atopic dermatitis: comparison with the IgE-mediated late-phase reaction. J Allergy Clin Immunol 1994;94:120-128.

-18 Kiehl P, Falkenberg K, Vogelbruch M, Kapp A: Tissue eosinophilia in acute and chronic atopic dermatitis: a morphometric approach using quantitative image analysis of immunostaining. Br J Dermatol 2001;145:720729.

19 Simon D, Braathen LR, Simon HU: Eosinophils and atopic dermatitis. Allergy 2004;59: 561-570.

20 Davis MD, Plager DA, George TJ, Weiss EA, Gleich GJ, Leiferman KM: Interactions of eosinophil granule proteins with skin: limits of detection, persistence, and vasopermeabilization. J Allergy Clin Immunol 2003;112: 988-994.

21 Kita, H, Adolphson CR, Gleich, GJ: Biology of eosinophils; in Middleton E Jr, Reed CE, Ellis EF, Adkinson NF Jr, Yunginger JW, Busse WW (eds): Allergy: Principles and Practice, ed. 6. St. Louis, Mosby-Yearbook Inc., 2003, pp 305-332.

22 Jacobsen EA, Taranova AG, Lee NA, Lee JJ: Eosinophils: singularly destructive effector cells or purveyors of immunoregulation? J Allergy Clin Immunol 2007;119:1313-1320.

23 Hossain M, Okubo Y, Sekiguchi M: Effects of various drugs (staurosporine, herbimycin A, ketotifen, theophylline, FK506 and cyclosporin A) on eosinophil viability. Arerugi 1994;43:711-717.

24 Hom JT, Estridge T: FK506 and rapamycin modulate the functional activities of human peripheral blood eosinophils. Clin Immunol Immunopathol 1993;68:293-300.

25 Kohyama T, Takizawa H, Kawasaki S, Akiyama N, Sato M, Ito K, Yamamoto K: A potent immunosuppressant FK506 inhibits IL-8 expression in human eosinophils. Mol Cell Biol Res Commun 1999;1:72-77.

-26 Hagan JB, Kita H, Gleich GJ: Inhibition of interleukin-5 mediated eosinophil viability by fluticasone 17-propionate: comparison with other glucocorticoids. Clin Exp Allergy 1998;28:999-1006.

-27 Horie S, Gleich GJ, Kita H: Cytokines directly induce degranulation and superoxide production from human eosinophils. J Allergy Clin Immunol 1996;98:371-381.
28 Busse W, Randlev B, Sedgwick J: The effect of azelastine on neutrophil and eosinophil generation of superoxide. J Allergy Clin Immunol 1989;83:400-405.

-29 Abu-Ghazaleh RI, Fujisawa T, Mestecky J, Kyle RA, Gleich GJ: IgA-induced eosinophil degranulation. J Immunol 1989;142:23932400.

30 Bankers-Fulbright JL, Kephart GM, Loegering DA, Bradford AL, Okada S, Kita H, Gleich GJ: Sulfonylureas inhibit cytokineinduced eosinophil survival and activation. J Immunol 1998;160:5546-5553.

31 Diggle PJ, Liang KY, Zeger SL: Analysis of Longitudinal Data. Oxford, Clarendon Press, 1994

32 Hultsch T, Kapp A, Spergel J: Immunomodulation and safety of topical calcineurin inhibitors for the treatment of atopic dermatitis. Dermatology 2005;211:174-187.

- 33 Inoue Y, Matsuwaki Y, Shin SH, Ponikau JU, Kita H: Nonpathogenic, environmental fungi induce activation and degranulation of human eosinophils. J Immunol 2005; 175 : 5439-5447.

-34 Bankers-Fulbright JL, Kephart GM, Bartemes KR, Kita H, O'Grady SM: Platelet-activating factor stimulates cytoplasmic alkalinization and granule acidification in human eosinophils. J Cell Sci 2004;117:57495757.

35 Kita H, Abu-Ghazaleh RI, Gleich GJ, Abraham RT: Role of pertussis toxin-sensitive G proteins in stimulus-dependent human eosinophil degranulation. J Immunol 1991;147: 3466-3473.

36 Giembycz MA, Lindsay MA: Pharmacology of the eosinophil. Pharmacol Rev 1999;51: 213-340.

- 37 Salo PM, Arbes SJ Jr, Sever M, Jaramillo R, Cohn RD, London SJ, Zeldin DC: Exposure to Alternaria alternata in US homes is associated with asthma symptoms. J Allergy Clin Immunol 2006;118:892-898.

- 38 O'Hollaren MT, Yunginger JW, Offord KP, Somers MJ, O'Connell EJ, Ballard DJ, Sachs MI: Exposure to an aeroallergen as a possible precipitating factor in respiratory arrest in young patients with asthma. $\mathrm{N}$ Engl J Med 1991;324:359-363.

-39 Scalabrin DM, Bavbek S, Perzanowski MS, Wilson BB, Platts-Mills TA, Wheatley LM: Use of specific IgE in assessing the relevance of fungal and dust mite allergens to atopic dermatitis: a comparison with asthmatic and nonasthmatic control subjects. J Allergy Clin Immunol 1999;104:1273-1279. 